\title{
THE RESPONSE OF SKIN FRICTION, WALL HEAT TRANSFER AND PRESSURE DROP TO WALL WAVINESS W THE PRESENCE OF BUOYANCY
}

\author{
C.N.B. RAO and K.S. SASTRI \\ Department of Mathematics \\ Indian Institute of Technology \\ Kharagpur-721 302 \\ INDIA
}

(Recelved in revised form November 5, 1981)

ABSTRACT. Laminar natural convection flow and heat transfer of a viscous incompressible fluid confined between two long vertical wavy walls has been analysed taking the fluid properties constant and variable. In particular, attention is restricted to estimate the effects of viscous dissipation and wall waviness on the flow and heat transfer characteristics. Use has been made of a linearization technique to simplify the governing equations and of Galerkin's method in the solution. The solutions obtained for the velocity and the temperature-fields hold good for all values of the Grashof number and wave number of the wavy walls.

KEY WORDS AND PHRASES. Fluid Mechanics, Incompressible viscous fluids, heat transfer, free convection, constant fluid properties (CFP), variable fluid properties (VFP). 1980 MATHEMATICS SUBJECT CLASSIFICATON CODES. 76099, 80A20, 76R10, 41A60, 41A10, $65 N 30$.

1. INTRODUCTION.

Vajravelu and Sastri [1,2] have reinvestigated Ostrach's work [3] of laminar natural convection flow and heat transfer of viscous incompressible fluids confined between two long vertical walls with a view to estimate the effect of wall-waviness on the flow and heat transfer characteristics in two cases, namely, (i) when one of the walls is wavy and (ii) when both the walls are wavy. The results of their analyses, however, seem to be meaningful for small values of the Grashof number only 
(the effect of viscous dissipation is likely to be negligible then!). But for large values of the Grashof number (definitely possible in many free convection studies), the assumption of constant fluid properties and neglect of viscous heating effects tend to be questionable. Indeed, Hong and Bergles [4] and Hwang et al. [5] among several others have pointed out that the assumption of constant fluid properties is chiefly responsible for large deviations between analytical predictions and experimental data.

The present work is therefore an attempt to re-examine the works mentioned in [1,2] when viscous heating effects are considered and when the fluid properties are both constant and variable. Approximate solutions of the governing equations have been obtained by Galerkin's method employing orthogonal polynomials which has proved to give results valid for all values of the wave number of the wavy walls and for all values of the Grashof number. With the help of these solutions, the flow and heat transfer characteristics have been evaluated numerically both when the fluid properties are constant and when they are variable. A comparative study of the results in the two cases when the viscous heating effects are taken into account has also been made; a detailed description of which has been given in $\S 5$.

\section{FORMULATION OF THE PROBLEM.}

Consider a channel made up of two long vertical wavy walls parallel to each other. Take the $\mathrm{X}$-axis vertically upward and the $\mathrm{Y}$-axis perpendicular to it in such a way that the wavy wall at the left may be represented by $\mathrm{Y}=-\mathrm{d}+\varepsilon^{*} \cos \lambda^{*} \mathrm{X}$ and the other wall by $Y=d+E \varepsilon^{*} \cos \lambda^{*} X$ (E, the ratio of the amplitudes of the wavy walls $=0(1))$. Let an infinite amount of viscous incompressible fluid be confined between the two walls which are at rest and maintained at two constant temperatures $\mathrm{T}_{\mathrm{WL}}$ and $\mathrm{T}_{\mathrm{WR}}$ respectively.

Under the assumptions that the flow is laminar, steady and two dimensional and that the fluid properties are temperature dependent the basic equations that govern the flow and heat transfer of the problem under consideration are given in Ostrach [3]. The boundary conditions are the well-known no-slip conditions of the velocity at both the walls and that the fluid temperature at the wall is equal to that of the wall. 
Making use of the dimensionless variables (refer to [3])

$x=\frac{X}{d}, y=\frac{Y}{d}, u=K \cdot \frac{U}{U_{R}}, v=K \cdot \frac{V}{U_{R}}, p=K G r \cdot \frac{P_{D}}{\rho_{O} U_{R}^{2}}, \theta=K \cdot \frac{T-T_{S}}{T_{W L}-T_{S}}$,

with $U_{R}=\frac{f \times \beta_{0}\left(T T_{W}-T_{S}\right) d^{2}}{\nu_{O}}$;

assuming that the fluid properties are temperature dependent (refer to [6]), and

using a linearization technique valid for small values of $\varepsilon(\varepsilon \ll 1$, refer to [1]),

we have reduced the governing equations into sets of ordinary differential equations for the mean parts $u_{0}, \theta_{0}$ and the perturbed parts $\psi, \emptyset$ as

$$
\begin{aligned}
& \left(1+\frac{A_{\mu}}{K} \theta_{0}\right) u_{0}^{\prime \prime}+\theta_{0}+\frac{A_{\mu}}{K} \theta_{0}^{\prime} \cdot u_{0}^{\prime}=c, \\
& \left(1+\frac{B_{k}}{K} \theta_{0}\right) \theta_{0}^{\prime \prime}+\frac{B_{k}}{K}\left(\theta_{0}^{\prime}\right)^{2}+\left(1+\frac{A_{\mu}}{K} \theta_{0}\right)\left(u_{0}^{\prime}\right)^{2}+K \propto=0, \\
& \left(1+\frac{A_{\mu}}{K} \theta_{0}\right)\left[\psi^{i v}-2 \lambda^{2} \psi^{\prime \prime}+\lambda^{4} \psi\right]-\emptyset^{\prime}-\frac{i \lambda G r}{K}\left[u_{0} \psi^{\prime \prime}-u_{0}^{\prime \prime} \psi-\lambda^{2} u_{0} \psi\right] \\
& +\frac{A_{\mu}}{K}\left[2 \theta_{0}^{\prime} \cdot \psi^{\prime \prime \prime}+\theta_{0}^{\prime \prime}\left(\psi^{\prime \prime}+\lambda^{2} \psi\right)-u_{0}^{\prime} \cdot\left(\emptyset^{\prime \prime}+\lambda^{2} \emptyset\right)-2 u_{0}^{\prime \prime} \cdot \emptyset^{\prime}-u_{0}^{\prime \prime \prime} \cdot \emptyset\right]=0 \text {, } \\
& \left(1+\frac{B_{k}}{K} \theta_{0}\right) \cdot\left(\emptyset^{\prime \prime}-\lambda^{2} \emptyset\right)-2\left(1+\frac{A_{\mu}}{K} \theta_{0}\right) u_{0}^{\prime} \cdot\left(\psi^{\prime \prime}+\lambda^{2} \psi\right)-\frac{i \lambda \operatorname{PrGr}}{K}\left(u_{0} \cdot \emptyset+\theta_{0}^{\prime} \psi\right) \\
& +\frac{B_{k}}{K}\left(2 \theta_{0}^{\prime} \cdot \emptyset^{\prime}+\theta_{0}^{\prime \prime} \cdot \emptyset\right)+{ }_{\mathrm{K}}^{A}\left(u_{0}^{\prime}\right)^{2} \emptyset=0 \text {, }
\end{aligned}
$$

where $\mathrm{C}\left(=\frac{\mathrm{dp}_{\mathrm{o}}}{\mathrm{dx}}\right)$ is taken as zero in the subsequent analysis, a prime denotes differentation with respect to $\mathrm{y}$, the functions $\psi, \emptyset$ are related to the perturbed parts $u_{1}, v_{1}, \theta_{1}$ through the relations

$$
\begin{aligned}
& u_{1}=-\frac{\partial \bar{\psi}}{\partial y}, \quad v_{1}=\frac{\partial \bar{\psi}}{\partial x}, \\
& \bar{\psi}=R 1 .\left\{\varepsilon e^{i \lambda x} \cdot \psi(y)\right\}, \quad \theta_{1}=R 1 .\left\{\varepsilon e^{i \lambda x} \cdot \emptyset(y)\right\},
\end{aligned}
$$

with $\mathrm{Rl}(\mathrm{F})$ denoting the real part of $\mathrm{F}$, and $\operatorname{Pr}\left(=\frac{\mu_{0} C_{p}}{k_{0}}\right)$ is the Prandt 1 number, $\operatorname{Gr}\left(=\frac{\mathrm{fx} \beta_{0}\left(T_{W L}-T_{S}\right) d^{3}}{\nu_{0}^{2}}\right)$ the Grashof number,

$\mathrm{K} \quad\left(=\operatorname{Pr} \bar{K}=\operatorname{PrGr} . \frac{\beta_{0} f x d}{C_{p}}\right)$ a dimensionless constant, 
$\propto\left(=\operatorname{PrGr} \bar{\alpha}=\frac{Q d^{2}}{k_{0}\left(T_{W L}-T_{S}\right)}\right)$ the heat source parameter,

$\mathrm{m}\left(=\frac{\mathrm{T}_{\mathrm{WR}}-\mathrm{T}_{\mathrm{S}}}{\mathrm{T}_{\mathrm{WL}}-\mathrm{T}_{\mathrm{S}}}\right)$ the wall-temperature ratio, and

$A\left(=a_{0}\left(T_{W L}-T_{0}\right)\right)$ and $B_{k}\left(=b_{0}\left(T_{W L}-T_{0}\right)\right)$ are dimensionless numbers describing the temperature-dependence of the fluid properties. (It is worth noting that, herein, the specific heat $C_{p}$ is taken constant as any changes in 1 t would be much smaller than $\varepsilon$ ).

The relevant boundary conditions on $u_{0}, \theta_{0}, \psi$ and $\emptyset$ are

$$
\begin{gathered}
u_{0}(-1)=0=u_{0}(+1), \quad \theta_{0}(-1)=\mathrm{K}, \quad \theta_{0}(+1)=\mathrm{mK}, \\
\psi(-1)=0=\psi(+1), \quad \psi^{\prime}(-1)=\mathrm{u}_{0}^{\prime}(-1), \quad \psi^{\prime}(+1)=E \mathrm{u}_{0}^{\prime}(+1), \\
\emptyset(-1)=-\theta_{0}^{\prime}(-1), \quad \emptyset(+1)=-E \theta_{0}^{\prime}(+1),
\end{gathered}
$$

3. SOLUTION OF THE MEAN PARTS $\left(u_{0}, \theta_{0}\right)$ AND THE PERTURBED PARTS $\left(u_{1}, v_{1}, \theta_{1}\right)$.

In this section we give a brief account of the approximate solutions obtained for the velocity- and the temperature-fields by the use of Galerkin's method employing orthogonal polynomials (refer to [8]).

As the equations $(2.2)-(2.3)$ are non-linear, we have rephrased them using an iteration scheme and taking $C$ equal to zero, as

$$
\begin{aligned}
& \left(1+\frac{A_{\mu}}{K} \theta_{o r-1}\right) u_{o r}^{\prime \prime}+\theta_{o r}+\frac{A_{\mu}}{K} \theta_{o r-1}^{\prime} \cdot u_{o r-1}^{\prime}=0, \\
& \left(1+\frac{B_{k}}{K} \theta_{o r-1}\right) \theta_{o r}^{\prime \prime}+\frac{B_{k}}{K}\left(\theta_{o r-1}^{\prime}\right)^{2}+\left(1+\frac{A_{\mu}}{K} \theta_{o r-1}\right)\left(u_{o r-1}^{\prime}\right)^{2}+k \alpha=0, \\
& \text { with } \quad u_{o r}(-1)=0=u_{o r}(+1), \theta_{o r}(-1)=k, \theta_{o r}(+1)=m k, r \geq 0, \\
& \text { and } u_{o r-1}^{\prime}=\theta_{o r-1}=\theta_{o r-1}^{\prime}=0 . \\
& \text { Approximating } u_{o r} \text { and } \theta_{o r} \text { as } \\
& u_{o r}(y)=\sum_{i=1}^{\infty} a_{i r} \cdot P_{i}(y), \theta_{o r}(y)=P_{o}(y)+\sum_{i=1}^{\infty} b_{i r} \cdot P_{i}(y),
\end{aligned}
$$

where $P_{0}(y)=\frac{K(m-1)}{2} \cdot y+\frac{K(m+1)}{2} \cdot y^{2}$,

$$
P_{1}(y)=1-y^{2}, \quad P_{2}(y)=y\left(1-y^{2}\right),
$$




$$
P_{3}(y)=\left(\frac{1}{7}-y^{2}\right)\left(1-y^{2}\right), \quad P_{4}(y)=y\left(\frac{1}{3}-y^{2}\right)\left(1-y^{2}\right) \text { and so on, (note }
$$

that $P_{1}(y)$ satisfy the non-homogeneous and homogeneous parts of the boundary conditions respectively when $1=0$ and when $i>0$ and further that $P_{1}(y)(i>0)$ are orthogonal to each other over the interval $-1 \leq y \leq 1$ ) and substituting them in equations (3.1) and (3.2) there result two residues: $r_{1}\left(y, a_{1 r}, b_{1 r}\right)$ and $r_{2}\left(y, a_{1 r}\right.$, $\left.b_{i r}\right)$. Orthogonalizing $r_{1}$ and $r_{2}$ with $P_{i}(i \geq 1)$ over the interval $-1 \leq$ y $\leq 1$ gives a set of linear equations for $a_{i}$ and $b_{i}$, solving which the values of the unknowns $a_{i}, b_{i}$ are determined. The foregoing method may be termed $G(p, q)$ method whenever a p-term approximation for $u_{\text {or }}$ and a q-term approximation for $\theta_{\text {or }}$ are employed.

Using $G(3,3)$ method, the constants $a_{i}$ and $b_{i}$ are determined directly when $r=0$ and iteratively when $\mathbf{r} \neq 0$, till a convergence criterion is met at $\mathbf{r}=\mathbf{r}_{\mathrm{c}}(\mathrm{say})$. (It has been observed that the $G(3,3)$ method gives results of considerable accuracy). The expressions (3.4) when $r=0$ and $r=r_{c}$ give the solutions for $u_{0}$ and $\theta_{0}$ in the absence of dissipation and in the presence of dissipation respectively. It may however be noted that the solutions $u_{0}, \theta_{0}$ correspond to the CFP-case when $A_{\mu}$ and $B_{k}$ are both zero and to the VFP-case when they are not zero.

In a similar way approximating $\psi$ and $\emptyset$ by two different orthogonal polynomials and using them in equations (2.4), (2.5) we have obtained solutions for them by G(3,3) method. Making use of these $\psi$ and $\emptyset$ in the relations $(2.6),(2.7)$ we got the expressions for the perturbed parts $u_{1}, v_{1}, \theta_{1}$.

\section{FLOW AND HEAT TRANSFER CHARACTERISTICS.}

As in reference [1], here too the shear stress, heat transfer coefficient and the pressure drop at the two walls are defined and non-dimensionalized. In view of the linearization technique [1] we write the shear stress $(T)$ and the heat transfer coefficient $(\mathrm{Nu})$ at either wall, in terms of mean and perturbed parts as

$$
T(x)=T^{0}+\varepsilon T^{1}(x), \quad N u(x)=N^{0}+\varepsilon N u^{1}(x),
$$

and the nondimensional pressure drop $\left(\mathrm{PD}^{1}\right)$ given as

$$
\mathrm{PD}_{(\mathrm{x})}^{1}=\frac{1}{\mathrm{KGr}}\left[\mathrm{p}_{1}(\mathrm{x}, 0)-\mathrm{p}_{1}(\mathrm{x}, \mathrm{y})\right]_{\mathrm{y}=\mathrm{yw}},
$$

with $p_{1}(x, y)=\int d p_{1}=\int\left(\frac{\partial p_{1}}{\partial x} d x+\frac{\partial p_{1}}{\partial y} d y\right)=$ perturbed pressure. 
With the help of the known expressions for $u_{0}, \theta_{0}$ and $u_{1}, v_{1}, \theta_{1}$, the expressions for the shear stress $(\mathrm{T})$, Nusselt number $(\mathrm{Nu})$ and pressure drop $\left(\mathrm{PD}^{1}\right)$ at both the walls have been found, but not presented here for the sake of brevity. The perturbed parts of the shear stress $\left(\mathrm{T}^{1}\right)$ and the Nusselt number $\left(\mathrm{Nu}^{1}\right)$ may be expressed in terms of their amplitudes and phases as

$$
\mathrm{T}_{L}^{1}(\mathrm{x})=\mathrm{AT}_{L}^{1} \cos \left(\lambda \mathrm{x}+\mathrm{PT}_{L}^{1}\right), \quad \mathrm{Nu}_{L}^{1}(\mathrm{x})=\mathrm{ANu}_{L}^{1} \cos \left(\lambda \mathrm{x}+\mathrm{PNu}_{L}^{1}\right),
$$

at the left wall and similarly those at the other wall. From the definition of the pressure drop, it may be observed that it indicates the difference of the pressure at any point $(x, y)$ in the flow field from that at the center of the channel.

Taking $\operatorname{Pr}=0.72$ (air), and given several sets of values to the parameters $K$, $\mathrm{m}, \propto, E, \mathrm{Gr}$ and $\lambda$, the expressions for the shear stresses, Nusselt numbers and pressure drops at the walls along with those of their amplitudes and phases have been evaluated numerically in the CFP-case when both $A_{\mu}$ and $B_{k}$ are zero and in the VFP-case when $A_{\mu}=0.0141405, B_{k}=0.0120485$ (fluid properties for air with $T_{0}=$ $\mathrm{T}_{\mathrm{S}}=15^{\circ} \mathrm{C}$ and $\mathrm{T}_{\mathrm{WL}}=20^{\circ} \mathrm{C}$, refer to [9] and also to [6]).

5. DISCUSSION OF THE RESULTS.

At the outset it is worth mentioning that the flow and heat transfer results for the dissipation case only have been presented in the figures 1 and 2 .

Figure 1 shows the mean velocity and mean temperature profiles in both the CFP- and VFP-cases when $\mathrm{m}=1$ and the parameters $\mathrm{K}, \propto$ take different values. From the figure it is clear that the results of the VFP-case are smaller in magnitude than those in the CFP-case, this behavior being more prominent for large values of $\mathrm{K}, \propto$ than for their small values.

Figure 2 describes the behaviors of the amplitudes of perturbed shear stresses and Nusselt numbers. From the figures $2(a)$ and $2(b)$ it is evident that the amplitudes of the former at both the walls increase with the wave number $\lambda$ and tend asymptotically to non-zero constant values. This asymptotic nature of the amplitudes holds good in the CFP- and VFP-cases as well as in the presence of viscous dissipation and in its absence. On a close look in to the figures $2(a), 2(b)$ it 

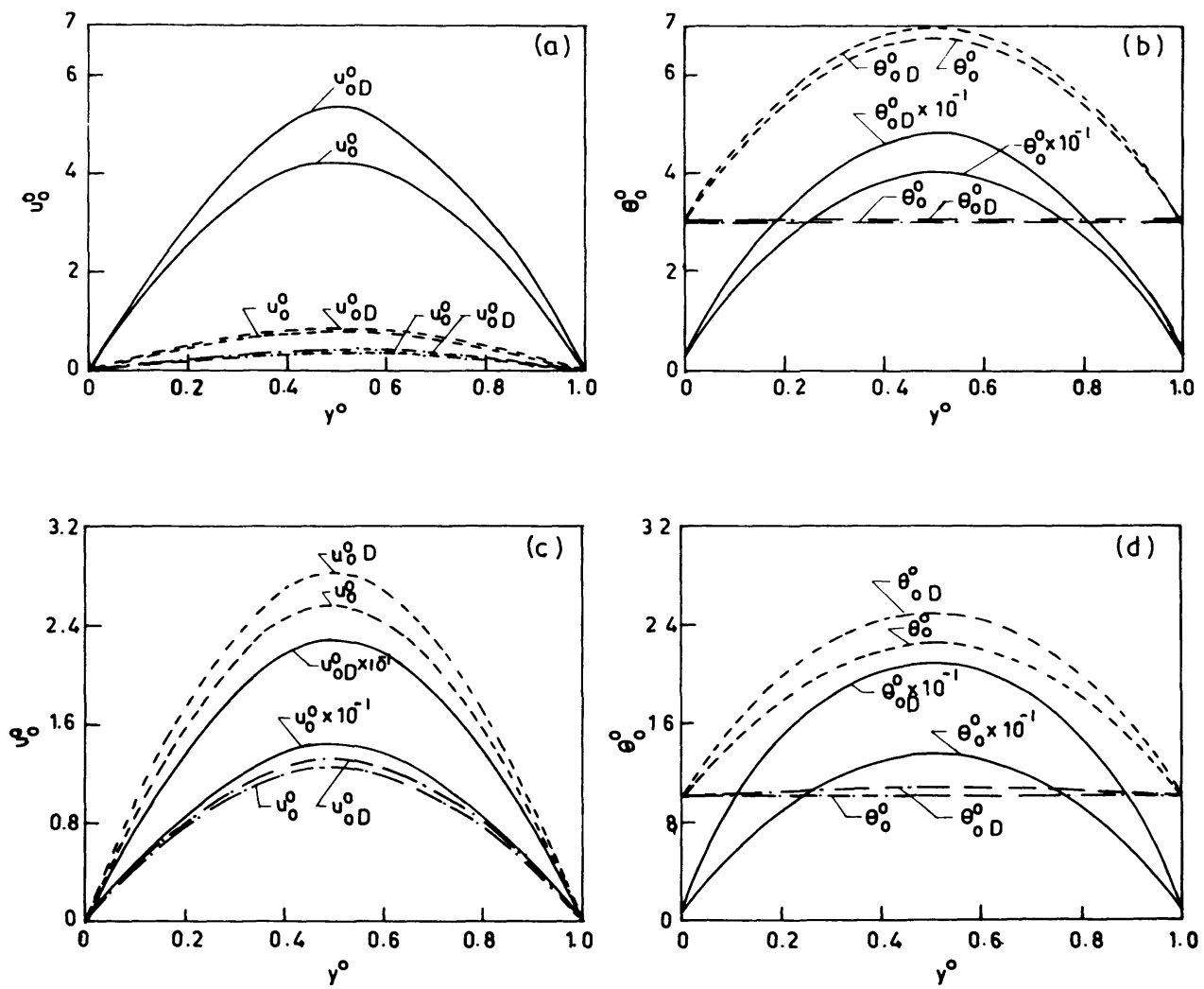

Figure 1. Mean velocity $\left(u_{0}^{o}\right)$ and mean temperature $\left(\theta_{0}^{\circ}\right)$ profiles $\left(u_{0}^{o}=4 u_{0}\right.$, $\left.\theta_{\mathrm{O}}^{\mathrm{O}}=16 \theta_{\mathrm{Q}}, \mathrm{K}^{\mathrm{O}}=16 \mathrm{~K}, \alpha^{\mathrm{O}}=4 \propto\right)$.
(a), (b): $\mathrm{K}^{\mathrm{O}}=3.0$,
(c), (d): $\mathrm{K}^{\mathrm{O}}=10.0$
m $\quad \alpha^{\mathrm{O}} \quad$ VFP $\quad$ CFP
$\begin{array}{llll}1.0 & 0.0 & \text { I } & 1\end{array}$
$\begin{array}{llll}1.0 & 10.0 & \text { II } & 2\end{array}$ 
becomes evident that the results of the VFP-case are smaller in magnitude than those of the CFP-case, this behavior becoming significant for large values of $\propto$ and Gr.

The amplitude-profiles of the perturbed Nusselt numbers shown in figures 2 (c), 2 (d) are similar in quality with those of the shear stresses in figures $2(a), 2(b)$ but in magnitude, the latter assume larger values than the former in almost all the cases. From the figures $2(\mathrm{c}), 2(\mathrm{~d})$ it may be further noticed that as in the case of the wall shear stresses, here too, the results of the VFP-case are, in general, smaller in magnitude than those of the CFP-case, this behavior becoming prominent when $\propto$ and $\mathrm{Gr}$ take large values.

From the numerical results it may be pointed out that (1) the effect of viscous dissipation is to increase the values of the amplitudes considerably and to enhance the effect of variable fluid properties, (2) the phases of the perturbed shear stress or perturbed Nusselt number at either wall are considerably smaller in magnitude than their amplitudes, and that they incresse for small values of $\lambda$ but decrease asymptotically to zero for its large values - a result holding good in the CFP- and VFP-cases, (3) the perturbed parts of shear stress, Musselt number and pressure drop at either wall are sinusoidal in nature (see expressions (4.1) for $T_{L}^{1}$ and $N_{L}^{1}$ ), and they are almost always positive for $0<\lambda x<\pi / 2$, become negative for $\pi / 2<\lambda \mathbf{x}<3 \pi / 2$, and take positive values again for $3 \pi / 2<\lambda \mathbf{x}<2 \pi$. This negative nature of shear stresses in the trough regions of the walls indicates physically that the perturbed flow tends to get separated there. Such instances are numerous because the wavy walls are infinitely long. However, this special feature of the perturbed flow which is of order $\varepsilon$, cannot persist in the total flow as this flow doesn't dominate the mean one. From the definition of the pressure drop and from its above-noted behavior it may be inferred that the fluid pressure at either wall lags behind (exceeds) that at the center of the channel whenever the pressure drops are positive (negative) in nature. Finally it way be concluded from the numerical computations that in the presence of dissipation the results of the VFP-case for large Grashof numbers ( $\mathrm{Gr}=100$, say) deviate from their comterparts of the CFP- 

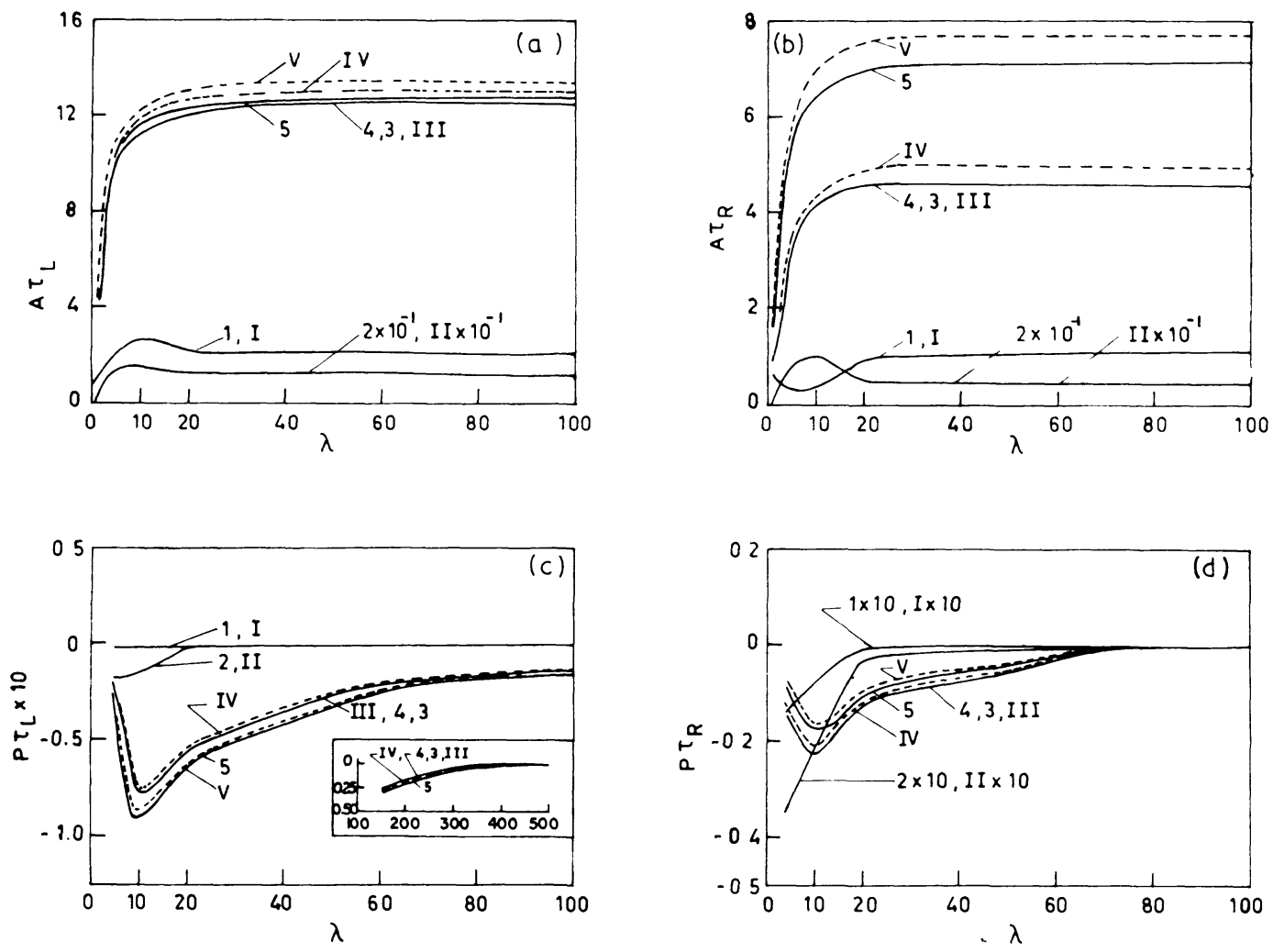

Figure 2. Amplitudes $\left(\mathrm{AT}_{\mathrm{L}}^{1}, \mathrm{Ar}_{\mathrm{R}}^{1}, \mathrm{ANu}_{\mathrm{L}}^{1}, \mathrm{ANu}_{\mathrm{R}}^{1}\right)$ of the perturbed shear stress and perturbed Nusselt number

$\begin{array}{lrll}\mathrm{K}^{\mathrm{O}}=10.0, & \mathrm{~m}=2.0, \quad \mathrm{E}=0.5 \\ \mathrm{c}^{\mathrm{O}} & \mathrm{Gr} & \mathrm{VFP} & \mathrm{CFP} \\ 0.0 & 5.0 & \mathrm{I} & 1 \\ 0.0 & 100.0 & \text { II } & 2 \\ 10.0 & 100.0 & \text { III } & 3\end{array}$


case by about 40 per cent in the case of iNusselt numbers and about 20 per cent in the case of wa11 shear stresses.

ACKNOWLEDGEMENTS.

The authors thank the referee for his valuable suggestions for the improvement of the work. They express their indebtedness to the RCC, Calcutta for the computer facilities and the I.I.T., Kharagpur for granting permission to one of the authors (C.N.B. Rao) to go to Calcutta to carry out the computations needed in this work. The former author (C.N.B. Rao) gratefully appreciates the financial assistance received from the CSIR, New Delhi, India.

\section{REFERENCES}

1. VAJRAVELU, K. and SASTRI, K.S. Free convective heat transfer of a viscous incompressible fluid confined between a long vertical wavy wall and a para11 lel flat wall. J. Fluid Mech. 86 (1978) 365-383.

2. Natural convective heat transfer in vertical wavy channels. Int. J. Heat Mass Transfer 23 (1980) 408-411.

3. OSTRACH, S. Laminar natural convection flow and heat transfer of fluids with and without heat sources in channels with constant wall temperatures. NACA Technical note no. 2863 (1952).

4. HONG, S.W. and BERGLES, A.E. Theoretical solutions for combined forced and free convection in horizontal tubes with temperature dependent viscosity. J. of Heat Transfer, ASME. 98 (1976) 1-7.

5. HWANG, S.T. and HONG, S.W. Effect of variable viscosity on laminar heat transfer in a rectangular duct. Chem. Eng. Progr. Symp. Ser. 66 (1970) 100-208.

6. VAJRAVELU, $K$. The effect of variable fluid properties on free convection flow of Afr/Water confined between two parallel vertical walls. Acta Mechanica 31 (1979) 199-211.

7. OSTRACH, S. New aspects of natural convection heat transfer preliminary study of effects of frictional heating. Trans. ASME. Paper No. 53-S-43 (January 1953) 1287-1290.

8. COLLATZ, L. (Ed.) The numerical treatment of differential equations ( $\$ 4$, Chapter 1). Springer-Verlag (1966).

9. BATCHELOR, G.K. (Ed.) An introduction to Fluid Dynamics (Appendix 1), Cambridge University Press, London (1967). 


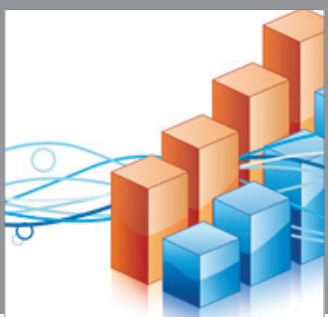

Advances in

Operations Research

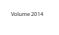

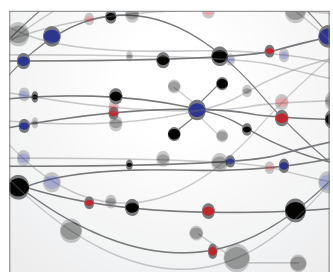

\section{The Scientific} World Journal
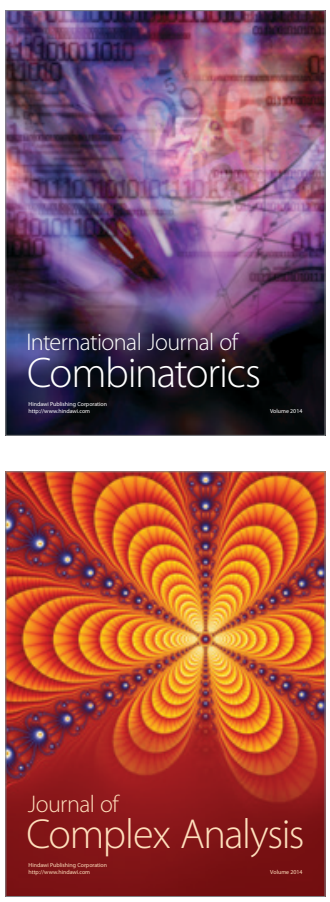

International Journal of

Mathematics and

Mathematical

Sciences
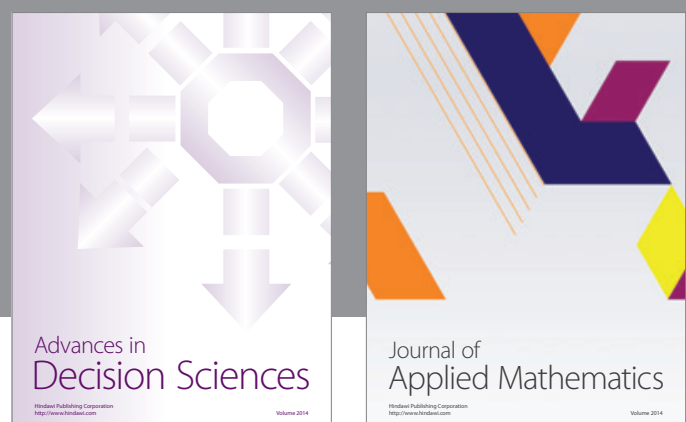

Journal of

Applied Mathematics
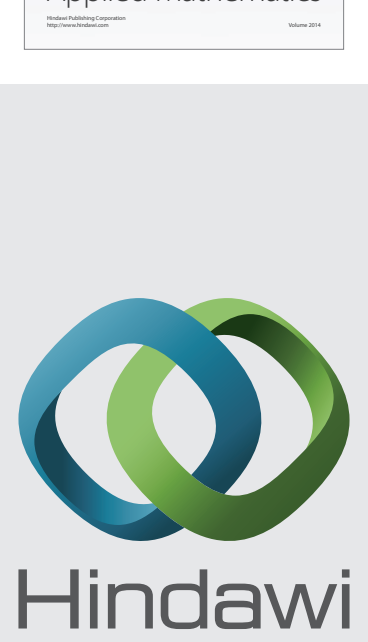

Submit your manuscripts at http://www.hindawi.com
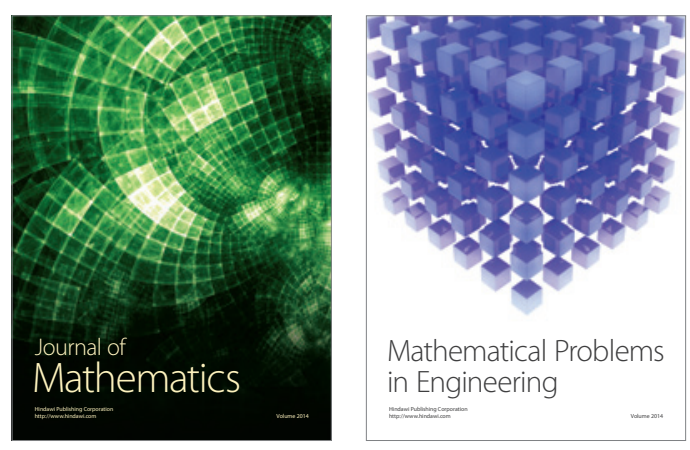

Mathematical Problems in Engineering
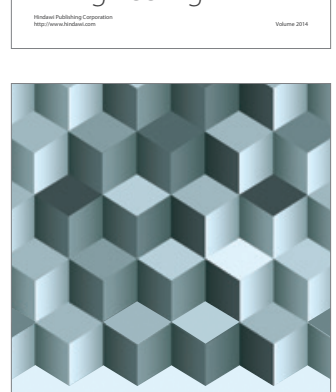

Journal of

Function Spaces
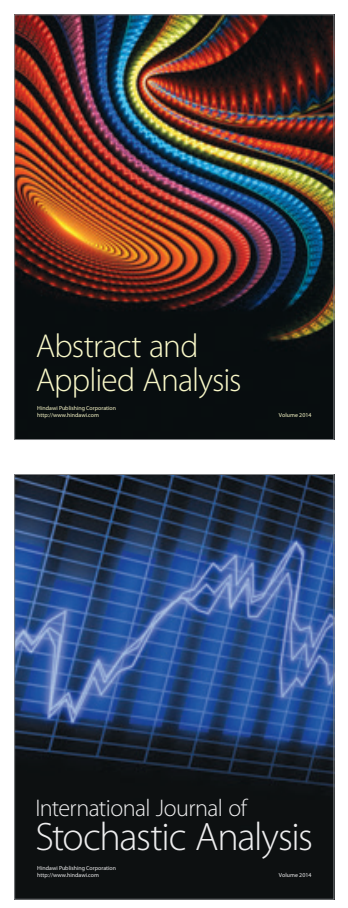

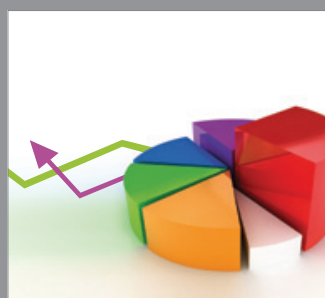

ournal of

Probability and Statistics

Promensencen
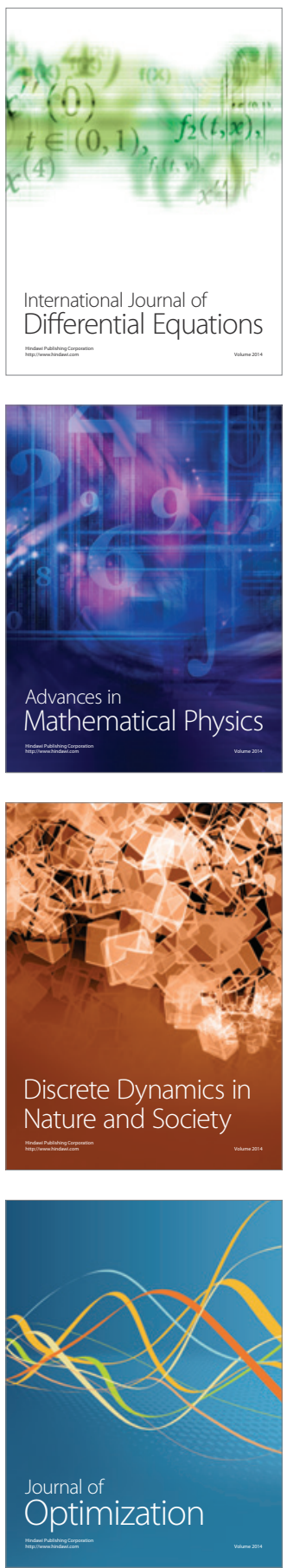